\title{
Caracterización química de panojas y vainas con semillas nativas del estado de Guerrero, México, para uso en la alimentación animal
}

\section{Chemical characterization of native panicles and seed pods of the State of Guerrero, Mexico, for use in animal nutrition}

\author{
Fernando Pérez-Gil Romoa, Ma. Elena Carranco Jáureguia, Ma. de la Concepción Calvo \\ Carrilloa, Lourdes Solanoa, Teresita de Jesús Martínez Iturbeb
}

\section{RESUMEN}

Se seleccionaron e identificaron cinco especies forrajeras nativas del estado de Guerrero, México: Bothriochloa sp, Sorghum halepense (L.) Pers, Desmanthus virgatus (L.) Willd, Gliricidia sepium (Jacq.) Stend, Acacia farnesiana (L.) Will, consideradas forrajes no convencionales por ser productos naturales, no utilizados actualmente para la alimentación animal. El objetivo fue determinar la composición química en muestras representativas de las cinco plantas, vainas con semillas y panojas. Los análisis realizados fueron: análisis químico proximal, fracciones de fibra, perfil de aminoácidos, factores antinutricios, minerales y vitaminas hidrosolubles. Se encontró que el valor de proteína cruda osciló entre 60 y 202, fibra cruda entre 132.5 y 354.8, extracto etéreo 17 a 32.4 , reportados en $\mathrm{g}^{-1} \mathrm{~kg}^{-1} \mathrm{de}^{-}$ materia seca (MS). El contenido de lignina en las gramíneas fue 52 a 82 y en las muestras de leguminosas fue de 132 a 202 g; mientras que la celulosa para el primer grupo fue de 189 a 276 y para el segundo de 95 a 269 g kg-1 MS. De los factores antinutricios analizados el inhibidor de tripsina cuantificado fue de 1.0 a 4.97 UIT mg-1, hemaglutininas y saponinas no se detectaron. Se analizaron los siguientes minerales: Ca (301-652), Mg (57-88), K (565-1286), $\mathrm{Na}$ (64.3-630), Zn (0.93-5.1) y Fe (8.3-21.0) (reportados en g kg-1). Se cuantificaron 17 aminoácidos y 3 vitaminas $\left(B_{1}, B_{2}\right.$ y $\left.B_{3}\right)$ de cada muestra presentando valores similares entre ellas. Se concluye que las panojas y las vainas con semillas podrían considerarse como un recurso no convencional en la alimentación de rumiantes.

PALABRAS CLAVE: Alimentos no convencionales, Especies forrajeras nativas, Composición química, Alimentación de rumiantes.

\begin{abstract}
We selected and identified five native forage plants of the State of Guerrero, México, considered unconventional fodder, not currently used for animal feed and having availability periods. The objective was to determine the chemical composition of representative samples of the five plants: seed pods from gramineas and panicles from legumes, as potential resources for ruminants. Analyses were proximal chemical, fiber fractions, amino acids profile, and nutritional factors, minerals, and water soluble vitamins. It was found that the crude protein value ranged between 60 to 202, crude fiber between 132.5 and 354.8, ether extract 17 to 32.4, reported in $\mathrm{g} \mathrm{kg}^{-1} \mathrm{DM}_{\text {. The lignin }}$ content was, in panicles 52-82 and in legume samples was 132-202 g, while the cellulose content was in the grasses 189-276 and in the legume samples was 95 to $269 \mathrm{~g}$. The antinutritional factors analyzed were trypsin inhibitor (1.0 to 4.97 UIT $\mathrm{mg}^{-1}$ ); saponins and hemagglutinins were not detected. The following minerals were analyzed: Ca (301652), Mg (57-88), K (565-1286), Zn (0.93-5.1) and Fe (8.3-21.0) (reported in $\mathrm{g} \mathrm{kg}^{-1}$ ). 17 amino acids were quantified and 3 hydrosolubles vitamins $\left(B_{1}, B_{2}\right.$ and $\left.B_{3}\right)$ of each sample with values similar to each other. The panicles and seed pods could be considered as an unconventional resource in ruminant feed.
\end{abstract}

KEY WORDS: Food unconventional native, Forage species, Chemical composition, Ruminant feeds.

Recibido el 14 de marzo de 2012. Aceptado el 19 de julio de 2013.

a Departamento de Nutrición Animal "Dr. Fernando Pérez-Gil Romo", Instituto Nacional de Ciencias Médicas y Nutrición Salvador Zubirán, Vasco de Quiroga No. 15, Col. Sección XVI, Delegación Tlalpan; 14000 México D.F. rexprimero@hotmail.com. Correspondencia al segundo autor.

b Facultad de Química, Universidad Nacional Autónoma de México. México, DF. 


\section{INTRODUCCIÓN}

La necesidad de buscar fuentes alternativas de alimento de buena calidad que resulten accesibles y de bajo costo para el consumo animal, ha traído como consecuencia la necesidad de realizar estudios sobre el potencial nutricional de alimentos que se encuentran disponibles en las diferentes regiones de la República Mexicana. Esta es un área de investigación y desarrollo de importancia a nivel mundial, debido a la conocida crisis de alimentos, a las restricciones por la baja producción agrícola y a la limitación de la agricultura para satisfacer la alta demanda de productos básicos por el incremento constante de la población. Esto eleva los costos de producción, ya que los granos, principales alimentos utilizados en la nutrición animal, son necesarios para la alimentación humana y se obtienen mayores ganancias al generar alimentos para consumo de la población, por lo cual surge la necesidad de evaluar productos con posibilidades de sustituir los ingredientes clásicos utilizados en la alimentación animal(1-3).

México cuenta con una gran diversidad de recursos alimenticios muy valiosos, su estudio brinda información para darles un mayor aprovechamiento y un beneficio para las comunidades más necesitadas de la región estudiada. Debido a la poca cantidad de agua en el subsuelo y la corta temporada de lluvias en el Municipio de Iguala al norte del estado de Guerrero, no es posible sembrar dos veces al año como sucede en otras regiones centrales del país, los productores ganaderos no tienen suficiente alimento (maíz, sorgo, etc.) durante el año, y se hace necesaria la compra de alimentos preparados comercialmente para alimentar al ganado, llevando como consecuencia un alto costo en la producción de estos(4). Por lo tanto, es necesario dar alternativas para la alimentación de rumiantes, utilizando alimentos no convencionales o no tradicionales, definiéndose estos como todo producto nativo u obtenido mediante un cultivo, o resultante de un producto primario, no

\section{INTRODUCTION}

The worldwide search for good quality, accessible, low-cost animal feeds is ongoing in response to the food crisis, limited input supply due to low agricultural production and the focus of agriculture on basic foods to feed the world's growing human population. These factors elevate costs because grains are the main elements in domestic animal nutrition but are also vital in the human diet. Indeed, food production for humans generates greater profits, highlighting the need to evaluate possible substitutes for traditional animal feed ingredients(1-3).

Mexico struggles with this dynamic, but has the advantage of a highly diverse flora offering resources for animal nutrition. More effective exploitation of these resources requires they be studied and that possible new animal feed sources be identified and developed. The Municipality of Iguala, in northern Guerrero State, Mexico, is an excellent example of the need to develop new feed sources to benefit local livestock producers. Its subsoils retain very little of the region's limited rainfall, making it impossible to sow grain crops twice a year, a situation common throughout central Mexico. Livestock producers therefore do not have sufficient feed reserves (e.g. corn, sorghum, etc.) to last the entire annual cycle and must purchase commercial feed for their animals. This substantially raises production costs(4). Under these circumstances, development of alternative feeds for ruminants is increasingly important. These use unconventional or non-traditional nutrient sources, defined as native products, crops or primary production by-products not currently used, or under-utilized, in animal nutrition. To be seen as an option in livestock systems, alternative feed ingredients must have a minimum availability in determined periods, provide one or more nutrients required by a cultured animal for normal development, and be innocuous in the administered forms and quantities(3). The present study objective was to describe the chemical composition of the 
utilizado actualmente o utilizado de manera escasa para la alimentación de animales, con un mínimo de disponibilidad en períodos determinados, el cual aporta uno o más de los nutrimentos requeridos por el organismo animal para su normal desarrollo, y que es inocuo en las formas y cantidades suministradas, representando una opción al problema de alimentación ganadera(3). Con base a lo anterior, el objetivo de este trabajo fue determinar la composición química de cinco plantas, vainas con semillas y panojas del estado de Guerrero, como posibles recursos para la alimentación de rumiantes.

\section{MATERIALES Y METODOS}

El área de estudio se ubicó en ejidos de la región del Valle de Iguala en Guerrero. La región presenta un clima cálido subhúmedo, escasa lluvia en verano y temperatura anual promedio de $26.7^{\circ} \mathrm{C}(5)$.

Las muestras colectadas cada semana se transportaron a la Ciudad de México para su secado en estufa para su posterior análisis químico. Este procedimiento se realizó en el periodo comprendido de febrero a marzo de 2008.

Las especies de estudio para este trabajo se eligieron observando y registrando la selección natural del ganado en libre pastoreo, además de la información recabada con los ganaderos. La recolección de las plantas se llevó a cabo de forma aleatoria y manualmente seleccionado aquéllas con una madurez grado medio. Para la identificación taxonómica de las muestras se tomaron las plantas completas (de raíz a flor) y se conservaron en hojas de papel; posteriormente se llevaron al Herbario Nacional del Instituto de Biología de la Universidad Nacional Autónoma de México.

Para el análisis químico se seleccionaron: para las gramíneas, raíz, tallo con hojas, flores y vainas con semillas; y para las leguminosas: raíz, tallo con hojas y panojas (espiga). El total de muestras colectadas fue de $20 \mathrm{~kg}$ de cada panicles of two grasses and the seedpods of three legume trees native to Guerrero state, Mexico, with a view to their possible use in ruminant feed systems.

\section{MATERIALS AND METHODS}

The study included local, communally-owned farms in the Iguala Valley, Guerrero state. Regional climate is subhumid warm with scarce summer rains and a $26.7^{\circ} \mathrm{C}$ annual average temperature(5).

Potential alternative feed plants were chosen for study by observing the selection process of free-grazing livestock and interviewing local ranchers. Collections were done weekly from February to March 2008. Plant selection was done randomly and manually, collecting individuals in a medium stage of maturity. Complete samples (root to flower) were collected, preserved and transported to the National Herbarium (Instituto de Biología, Universidad Nacional Autónoma de México) for identification.

Samples for chemical analysis included roots, stems with leaves, flowers and pods with seeds for legumes, and roots, stems with leaves and panicles (spikes) for grasses. During the collection period, total samples of $20 \mathrm{~kg}$ per species were collected. Plant material was manually cleaned to remove material unrelated to the study, leaving only panicles (grasses) and seedpods (legumes). For each species, the samples collected at different locations were pooled to create a single overall sample per species. These were transported weekly to the laboratory (Departamento de Nutrición Animal "Dr. Fernando Pérez-Gil Romo", Instituto Nacional de Ciencias Médicas y Nutrición Salvador Zubirán) for analysis. Samples were dried in a convection oven at $60{ }^{\circ} \mathrm{C}$ for $24-48 \mathrm{~h}$, ground in a hammer mill at $650 \mathrm{rpm}$ and sieved through a $1 \mathrm{~mm}$ diameter mesh. The resulting flours were stored in separate containers in a cool, dark place until use.

For each species, proximate analyses were run following standard AOAC techniques(6): moisture 
especie comprendida en el periodo de los meses mencionados.

Todas las plantas se limpiaron en forma manual, eliminando material ajeno al estudio, y quedando para las gramíneas las panojas, y para las leguminosas vainas con semillas. La colecta de cada especie en los diferentes sitios de los ejidos se mezclaron para tener una muestra única. Posteriormente se transportaron al Departamento de Nutrición Animal "Dr. Fernando Pérez-Gil Romo" del Instituto Nacional de Ciencias Médicas y Nutrición Salvador Zubirán para sus análisis. Se secaron en una estufa de convección a $60^{\circ} \mathrm{C} /$ 24-48 $\mathrm{h}$, se molieron utilizando un molino de martillos a $650 \mathrm{rpm}$ con una criba de $1 \mathrm{~mm}$ de diámetro de partícula. Las harinas se guardaron en frascos en lugar fresco y libre de luz.

De cada especie seleccionada se analizaron químicamente por las técnicas estandarizadas descritas en AOAC(6): humedad (método 934.01), cenizas (método 942.05), proteína cruda
(934.01); ash (942.05); crude protein ( $\mathrm{N} \times 6.25)$ (976.05); ether extract (920.39); crude fiber (962.09); and nitrogen-free extract (soluble carbohydrates) by difference. Following the methods of Van Soest(7), six fiber fractions were quantified: neutral detergent fiber (NDF); acid detergent fiber (ADF); lignin; cellulose; hemicellulose; and silica. Antinutrients identified in the samples were trypsin inhibitors(8); hemagglutinins $(9)$; and saponins (qualitative test)(10). Other analyses included minerals by atomic absorption spectrophotometry(6), amino acid profile (Waters Acc-QTAG, 1993), thiamin (953.17), riboflavin (970.65) and niacin $(975.41)^{(6)}$. All data were analyzed with quantitative descriptive statistics (PASW Statistics, v. 18, 2009).

\section{RESULTS}

Five potential alternative feed plants were identified in the collection: two grasses

Cuadro 1. Análisis químico aproximado de panojas de gramíneas y vainas con semillas de leguminosas $\left(\mathrm{g} \mathrm{kg}^{-1}\right.$ de muestra)

Table 1. Chemical composition of the panicles of two wild grasses and the seedpods of three wild legumes $\left(\mathrm{g} \mathrm{kg}^{-1}\right.$ sample)

\begin{tabular}{lcccccc}
\hline & Moisture & Ash & Crude protein & Ether extract & Crude fiber & NFE $^{2}$ \\
\hline Grasses: & & & & & & \\
Bothriochloa sp. & 18 & 88 & 60 & 23 & 277 & 534 \\
CV & 0.12 & 0.01 & 0.39 & 0.01 & 0.01 & \\
Sorghum halepense (L.) Pers & 28 & 82 & 82 & 24 & 192 & 593 \\
CV & 0.01 & 0.03 & 0.03 & 0.08 & 0.05 & \\
Legumes: & & & & & & \\
Desmanthus virgatus (L.) Willd & 34 & 62 & 202 & 19 & 212 & 473 \\
CV & 0.01 & 0.03 & 0.03 & 0.08 & 0.05 & \\
Gliricidia sepium (Jacq.) Stend & 47 & 44 & 132 & 32 & 355 & 389 \\
CV & 0.01 & 0.03 & 0.009 & 0.05 & 0.02 & \\
Acacia farnesiana (L.) Willd & 47 & 45 & 169 & 17 & 133 & 590 \\
CV & 0.004 & 0.01 & 0.03 & 0.05 & 0.01 & \\
\hline
\end{tabular}

$\mathrm{n}=6$ replicates.

$1 \mathrm{~N} \times 6.25$.

2 Nitrogen-free extract, by difference.

$\mathrm{CV}=$ Coefficient of variation. 
( $\mathrm{N} \times$ 6.25) (método 976.05), extracto etéreo (método 920.39), fibra cruda (método 962.09) y extracto libre de nitrógeno (carbohidratos solubles) por diferencia. Fracciones de fibra por los métodos descritos por Van Soest(7): fibra detergente neutro (FDN), fibra detergente ácido (FDA), lignina, celulosa, hemicelulosa y sílice. Factores antinutricios: inhibidor de tripsina(8), hemaglutininas (prueba cualitativa)(9), saponinas (prueba cualitativa)(10), minerales por espectrofotometría de absorción atómica(6), perfil de aminoácidos (Waters Acc-QTAG, 1993) y vitaminas: tiamina (método 953.17), riboflavina (método 970.65) y niacina (método 975.41)(6).

Se llevó a cabo un análisis estadístico descriptivo cuantitativo (PASW Statistics, v. 18, 2009).

\section{RESULTADOS}

Se identificaron taxonómicamente dos especies forrajeras de la familia de las gramíneas:
(Bothriochloa sp. and Sorghum halepense (L.) Pers); and three legumes (Desmanthus virgatus (L.) Willd, Gliricidia sepium (Jacq.) Stend and Acacia farnesiana (L.) Willd).

Proximate composition data were expressed as the means $\left(\mathrm{g}^{-1} \mathrm{~kg}\right)$ for each studied variable for grasses and legumes. In the grasses, ash content was 85, crude protein ranged from 60 to 82 , ether extract was 23 and crude fiber ranged from 192 to 277. In the legumes, average ash content ranged from 44 to 62, crude protein from 132 to 202, ether extract from 17 to 32, and crude fiber from 133 to 355 (Table 1).

The fiber fractions were expressed as grams per kilogram dry matter ( $\mathrm{g} / \mathrm{kg} \mathrm{DM})$ (Table 2). In the grasses, NDF (cell wall) content was 614 to 689 , ADF was 293 to 341 , lignin was 53 to 84 , cellulose was 194 to 281 , hemicellulose was 321 to 348 and silica was 6 to 20 . The legumes

Cuadro 2. Fracciones de fibra de panojas de gramíneas y vainas con semillas de leguminosas $\left(\mathrm{g} \mathrm{kg}^{-1}\right.$ de muestra seca)

Table 2. Fiber fractions of grass panicles and legume seed pods ( $\mathrm{g} \mathrm{kg}^{-1}$ dry matter)

\begin{tabular}{lcccccc}
\hline & NDF & ADF & & & & \\
& (cell walls) & (cell content) & Lignin & Cellulose Hemicellulose & Silica \\
\hline Grasses: & & & & & & \\
Bothriochloa sp. & 689.41 & 341.14 & 52.95 & 281.06 & 348.27 & 6.11 \\
CV & 0.003 & 0.01 & 0.10 & 0.005 & 0.009 & 0.12 \\
Sorghum halepense (L.) Pers & 614.20 & 293.21 & 84.36 & 194.44 & 320.99 & 19.55 \\
CV & 0.005 & 0.01 & 0.04 & 0.03 & 0.007 & 0.02 \\
Legumes: & & & & & & \\
Desmanthus virgatus (L.) Willd & 460.66 & 254.66 & 209.11 & 177.02 & 206.00 & 3.11 \\
CV & 0.01 & 0.01 & 0.02 & 0.01 & 0.02 & 0.18 \\
Gliricidia sepium (Jacq.) Stend & 587.62 & 417.63 & 138.51 & 282.27 & 169.99 & 5.25 \\
CV & 0.01 & 0.01 & 0.03 & 0.01 & 0.01 & 0.17 \\
Acacia farnesiana (L.) Willd & 238.20 & 162.64 & 177.33 & 99.69 & 75.55 & 4.20 \\
CV & 0.01 & 0.01 & 0.17 & 0.09 & 0.03 & 0.25 \\
\hline
\end{tabular}

$\mathrm{n}=6$ replicates.

NDF $=$ Neutral detergent fiber.

ADF $=$ Acid detergent fiber.

$\mathrm{CV}=$ Coefficient of variation 
Bothriochloa sp, Sorghum halepense (L.) Pers y tres de leguminosas: Desmanthus virgatus (L.) Willd, Gliricidia sepium (Jacq.) Stend, Acacia farnesiana (L.) Willd.

Análisis químico. Del análisis de medias de las variables estudiadas (se reportan en gramos por kilogramo) para la composición química de las gramíneas, en promedio, fue: cenizas 85, proteína cruda los valores estuvieron entre 60 y 82 , extracto etéreo de 23 y fibra cruda entre 192 y 277. Para las leguminosas estudiadas se encontró que el contenido de cenizas se mantuvo entre 44 y 62, para proteína cruda 132 a 202, extracto etéreo de 17 a 32, mientras que fibra cruda de 133 a 355 (Cuadro 1).

En cuanto a las fracciones de fibra (Cuadro 2) (se reportan en gramos por kilogramos de materia seca) las gramíneas presentan el mayor contenido de FDN o paredes celulares (614 a 689), para FDA (293 a 341), lignina (53 a 84), celulosa (194 a 281), hemicelulosa (321a 348) y sílice (6 a 20). Con respecto a las leguminosas FDN (238 a 588), FDA (162 a 417), lignina (138 a 209), celulosa (100 a 282), hemicelulosa (76 a 206) y sílice (3 a 5).
Cuadro 3. Factores antinutricios de panojas de gramíneas y vainas con semillas de leguminosas

Table 3. Antinutritional factors in grass panicles and legume seed pods

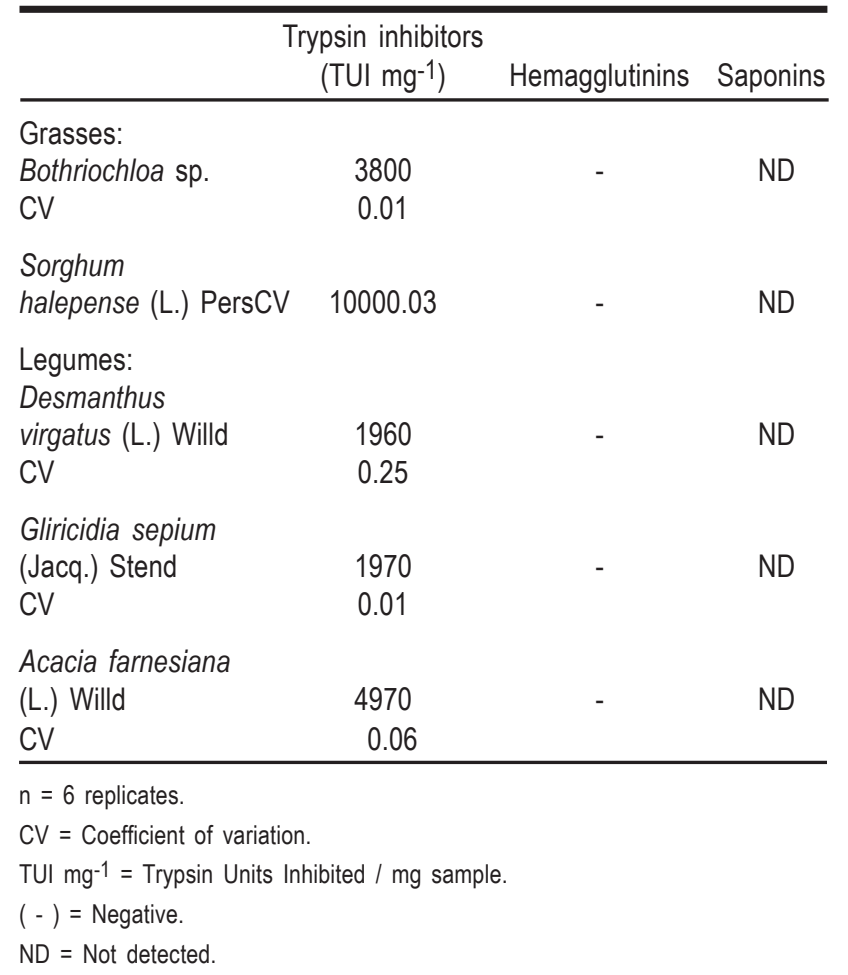

Cuadro 4. Resultados de minerales de las panojas de gramíneas y vainas con semillas ( $\mathrm{g} \mathrm{kg}^{-1}$ de muestra) Table 4. Mineral content of grass panicles and legume seedpods ( $\mathrm{g} \mathrm{kg}^{-1}$ sample)

\begin{tabular}{lcccccc}
\hline & Calcium & Magnesium & Potassium & Sodium & Zinc & Iron \\
\hline Grasses: & & & & & & \\
Bothriochloa sp. & 360.5 & 61.8 & 623.6 & 630 & 5.1 & 18.5 \\
CV & 0.007 & 0.004 & 0.03 & 0.31 & 0.002 & 0.05 \\
Sorghum halepense (L.)Pers. & 321.9 & 88.9 & 565.4 & 118.0 & 4.7 & 21.2 \\
CV & 0.01 & 0.004 & 0.02 & 0.05 & 0.02 & 0.09 \\
Legumes: & & & & & & \\
Desmanthus virgatus (L.) Willd & 302.10 & 57.3 & 1079.3 & 80.3 & 0.93 & 8.7 \\
CV & 0.04 & 0.02 & 0.01 & 0.001 & 0.04 & 0.01 \\
Gliricidia sepium (Jacq.) Stend & 336.3 & 61.3 & 1286.8 & 90.6 & 1.1 & 8.8 \\
CV & 0.03 & 0.04 & 0.05 & 0.08 & 0.002 & 0.20 \\
Acacia farnesiana (L.) Willd & 652.0 & 71.2 & 898.9 & 64.3 & 2.2 & 8.3 \\
CV & 0.01 & 0.05 & 0.009 & 0.18 & 0.14 & 0.04 \\
\hline
\end{tabular}

$\mathrm{n}=6$ replicates.

$\mathrm{CV}=$ Coefficient of variation . 
CARACTERIZACIÓN QUÍMICA DE ESPECIES FORRAJERAS NATIVAS PARA USO EN LA ALIMENTACIÓN ANIMAL

Los valores reportados para el inhibidor de tripsina en gramíneas fueron de 1,000 a 3,800 y en leguminosas de 1,960 a 4,970 UIT mg-1. En ambos grupos de muestras la prueba de hemaglutininas dio negativa, y no se detectaron saponinas (Cuadro 3).

En las gramíneas el contenido de minerales (gramos por kilogramo) fue: Ca (322 a 360), Mg (62 a 89), K (565 a 623), Na (565 a 630), Zn (4.7 a 5.1$)$ y Fe (18 a 21$)$ y en leguminosas: Ca (302 a 652), Mg (57 a 71), K (899 a 1287), $\mathrm{Na}(64$ a 91$), \mathrm{Zn}(0.9$ a 2.2$)$ y Fe (8.3 a 8.8) (Cuadro 4).

Se reporta el contenido de 8 aminoácidos esenciales (Cuadro 5) y 9 no esenciales (Cuadro 6) y tres vitaminas hidrosolubles (Cuadro 7) siendo similares los resultados tanto para gramíneas como para leguminosas.

\section{DISCUSIÓN}

Las plantas identificadas taxonómicamente fueron las gramíneas: Bothriochloa sp (pasto had a NDF content ranging from 238 to 588, an ADF content from 162 to 417, lignin from 138 to 209, cellulose from 100 to 282, hemicellulose from 76 to 206 and silica from 3 to 5 .

Of the three analyzed antinutrients, trypsin inhibitors were present in the grasses $(1,000$ to 3,800 TUI $\mathrm{mg}^{-1}$ ) and the legumes (1,960 to 4,970 TUI $\mathrm{mg}^{-1}$ ). The hemagglutinins test was negative in both groups and saponins were undetected (Table 3).

Mineral content $\left(\mathrm{g}^{-1} \mathrm{~kg}\right)$ in the grasses was 322 to 360 for Ca; 62 to 89 for Mg; 565 to 623 for $\mathrm{K}$; 565 to 630 for $\mathrm{Na}$; 4.7 to 5.1 for $\mathrm{Zn}$; and 18 to 21 for Fe. In the legumes, the mineral contents were 302 to 652 for $\mathrm{Ca} ; 57$ to 71 for $\mathrm{Mg} ; 899$ to 1287 for K; 64 to 91 for $\mathrm{Na} ; 0.9$ to 2.2 for $\mathrm{Zn}$; and 8.3 to 8.8 for Fe (Table 4 ).

Both the grasses and the legumes contained similar levels of 8 essential and 9 non-essential amino acids (Tables 5 and 6 ), and hydrosoluble vitamins (Table 7).

Cuadro 5. Perfil de aminoácidos (aa) esenciales de panojas de gramíneas y vainas con semillas de leguminosas ( $\mathrm{g}$ de aa/100 g de proteína)

Table 5. Essential amino acids (aa) profile of grass panicles and legume seedpods (g aa/100 g protein)

\begin{tabular}{lcccccccc}
\hline & Ile & Leu & Lys & Met & Pha & Thr & Val & His \\
\hline Grasses: & & & & & & & & \\
Bothriochloa sp. & 2.6 & 7.6 & 1.9 & 2.3 & 3.2 & 3.0 & 4.3 & 1.5 \\
CV & 0.007 & 0.003 & 0.005 & 0.004 & 0.01 & 0.01 & 0.002 & 0.01 \\
Sorghum halepense (L.) Pers & 2.5 & 7.4 & 1.9 & 2.3 & 3.1 & 3.0 & 4.3 & 1.6 \\
CV & 0.01 & 0.001 & 0.0 & 0.004 & 0.006 & 0.009 & 0.002 & 0.006 \\
Legumes: & & & & & & & & \\
Desmanthus virgatus (L.) Willd & 3.5 & 12 & 2.3 & 2.4 & 5.0 & 3.8 & 5.2 & 2.2 \\
CV & 0.002 & 0.001 & 0.004 & 0.01 & 0.001 & 0.002 & 0.007 & 0.004 \\
Gliricidia sepium (Jacq.) Stend & 2.6 & 8.1 & 2.1 & 2.3 & 4.6 & 3.2 & 4.6 & 1.6 \\
CV & 0.003 & 0.001 & 0.009 & 0.01 & 0.002 & 0.003 & 0.006 & 0.01 \\
Acacia farnesiana (L.) Willd & 3.4 & 12 & 2.2 & 2.3 & 4.9 & 3.4 & 4.9 & 2.0 \\
CV & 0.002 & 0.002 & 0.009 & 0.008 & 0.004 & 0.008 & 0.004 & 0.009 \\
\hline
\end{tabular}

$\mathrm{n}=2$ replicates.

$\mathrm{CV}=$ Coefficient of variation . 
Cuadro 6. Perfil de aminoácidos (aa) no esenciales de panojas de gramíneas y vainas con semillas de leguminosas (g de aa/100 g de proteína)

Table 6. Non-essential amino acids (aa) profile of grass panicles and legume seedpods ( $\mathrm{g}$ aa/100 $\mathrm{g}$ protein)

\begin{tabular}{lccccccccc}
\hline & Cys & Tyr & Arg & Ala & Asp & Glu & Gly & Pro & Ser \\
\hline Grasses: & & & & & & & & & \\
Bothriochloa sp. & 2.9 & 3.2 & 3.3 & 6.5 & 5.5 & 12.4 & 3.1 & 8.8 & 4.0 \\
CV & 0.003 & 0.009 & 0.005 & 0.004 & 0.003 & 0.003 & 0.003 & 0.001 & 0.002 \\
Sorghum halepense (L.) Pers & 2.8 & 2.3 & 3.3 & 6.5 & 5.6 & 12.4 & 3.1 & 8.8 & 4.1 \\
CV & 0.007 & 0.01 & 0.006 & 0.003 & 0.005 & 0.008 & 0.0 & 0.002 & 0.002 \\
Legumes: & & & & & & & & & \\
Desmanthus virgatus (L.) Willd & 3.5 & 3.4 & 3.9 & 6.9 & 6.9 & 14.6 & 3.1 & 10.3 & 4.2 \\
CV & 0.002 & 0.005 & 0.002 & 0.001 & 0.002 & 0.002 & 0.01 & 0.003 & 0.004 \\
Gliricidia sepium (Jacq.) Stend & 3.0 & 3.5 & 3.4 & 6.5 & 5.9 & 13.5 & 3.1 & 8.8 & 4.1 \\
CV & 0.003 & 0.005 & 0.005 & 0.004 & 0.005 & 0.002 & 0.003 & 0.005 & 0.002 \\
Acacia farnesiana (L.) Willd & 3.4 & 3.7 & 3.7 & 6.5 & 6.7 & 13.5 & 3.1 & 10.1 & 4.1 \\
CV & 0.008 & 0.005 & 0.008 & 0.003 & 0.001 & 0.002 & 0.003 & 0.001 & $<0.002$ \\
\hline
\end{tabular}

$\mathrm{n}=2$ replicates.

$\mathrm{CV}=$ Coefficient of variation.

barbados), perenne de 30 a $50 \mathrm{~cm}$ de alto, crece en zonas áridas, es resistente al pastoreo intensivo y a sequías breves y se utiliza como forraje. Sorghum halepense (L.) Pers (zacate o pasto Johnson), perenne de 50 a $200 \mathrm{~cm}$ de alto. Invade todo terreno y es difícil de erradicar. Se puede ensilar como alimento para el ganado. Las leguminosas identificadas fueron: Desmanthus virgatus (L.) Willd, planta arbustiva con tallos de más de $2 \mathrm{~m}$ de alto, crece en suelos arenosos de textura frágil, así como en suelos arcillosos, se emplea como forraje para ramoneo y se considera, por su contenido de proteína cruda, semejante a la alfalfa. Gliricidia sepium (Jacq.) Stend (mataratón o madre del cacao) arbusto de 10 a $15 \mathrm{~m}$ de altura con un crecimiento rápido, resistente al ramoneo y puede utilizarse como poste para cercas a lo largo de praderas, llegándose a podar a una altura de $1.5 \mathrm{~m}$ para que los animales la alcancen. Acacia farnesiana (L.) Willd (mimosa), arbusto espinoso que crece rápidamente, sus hojas son una excelente fuente de ramoneo aunque se considera como una planta con un alto contenido de taninos(11-16).
Cuadro 7. Contenido de vitaminas en panojas de gramíneas y vainas con semillas de leguminosas $\left(\mathrm{g} \mathrm{kg}^{-1}\right.$ de muestra)

Table 7. Vitamin content of grass panicles and legume seed pods ( $\mathrm{g} \mathrm{kg}^{-1}$ sample)

\begin{tabular}{lccc}
\hline & Thiamin & Riboflavin & Niacin \\
\hline Grasses: & & & \\
Bothriochloa sp. & 0.65 & 1.2 & 1.9 \\
CV & 0.02 & 0.01 & 0.007 \\
Sorghum halepense (L.) Pers & 0.79 & 1.2 & 1.9 \\
CV & 0.01 & 0.01 & 0.007 \\
Legumes: & & & \\
Desmanthus virgatus (L.) & & & \\
Willd & 0.815 & 1.1 & 2.0 \\
CV & 0.008 & 0.008 & 0.003 \\
Gliricidia sepium (Jacq.) & & & \\
Stend & 0.893 & 1.2 & 1.8 \\
CV & 0.004 & 0.01 & 0.007 \\
Acacia farnesiana (L.) Willd & 0.905 & 1.3 & 1.8 \\
CV & 0.007 & 0.01 & 0.01 \\
\hline $\mathrm{n}=2$ replicates. & & & \\
CV = Coefficient of variation. & & & \\
\end{tabular}


Los forrajes se producen principalmente para la alimentación del ganado, por lo que es importante conocer los factores que afectan su valor nutricional. Desde el punto de vista de las aplicaciones prácticas el valor nutricional del forraje depende principalmente de su contenido de nutrimentos solubles (proteínas, carbohidratos y minerales), así como del grado de disponibilidad de estos nutrimentos(17).

Análisis químico. Las muestras de gramíneas tuvieron un mayor contenido de cenizas que las leguminosas. Ramírez et al(18) analizaron muestras del noroeste del país encontrando un contenido de cenizas mayor (9.1 a $12.1 \%$ ) a lo reportado en este estudio. Al identificar los componentes de esta fracción se encontró que en el caso de las gramíneas los valores de cada mineral fueron similares, mientras que en las leguminosas la muestra de Acacia farnenciana (L.) Willd tuvo el doble de Ca y Zn, de K tuvo el menor contenido así como de $\mathrm{Na}$ y de $\mathrm{Mg}$ presentó la mayor concentración. Las otras dos muestras de leguminosas mostraron datos similares.

Un factor importante que influye en el contenido de cenizas y minerales es la fertilidad de los suelos y el desarrollo de la planta. En general los forrajes producidos bajo condiciones adecuadas de fertilización contienen una cantidad suficiente de estas fracciones para satisfacer las necesidades del ganado. El deterioro del ganado se puede presentar a pesar de tener abundancia de alimento; esto se puede deber a una deficiencia o exceso de minerales en los forrajes, provocando problemas de baja producción y reproducción entre los rumiantes en pastoreo. Se ha reportado que la deficiencia de minerales además, puede provocar extenuación, pérdida de pelo, desórdenes de la piel, abortos no infecciosos, diarrea, anemia, pérdida de apetito, anormalidades óseas, baja fertilidad y pérdida de peso, entre otros signos clínicos(19).

En cuanto a las gramíneas, se observa un contenido de proteína cruda (PC) bajo, esto

\section{DISCUSSION}

Taxonomic analysis identified two grass species grazed by livestock in the study area. Bothriochloa sp. (Barbados grass) is a perrenial grass 30 to $50 \mathrm{~cm}$ in height, that grows in arid zones, resists intensive grazing and brief droughts, and is used as forage. Sorghum halepense (L.) Pers (Johnson grass) is a perrenial 50 to $200 \mathrm{~cm}$ in height known for invading any type of terrain and being difficult to eradicate; it can be ensilaged as cattle feed. Three legumes were identified. Desmanthus virgatus (L.) Willd is a bushy plant with stems taller than $2 \mathrm{~m}$ in height that grows in sandy, fragile and clayey soils. It is used as browse for livestock and has a crude protein content similar to that of alfalfa. Gliricidia sepium (Jacq.) Stend is a bush 100 to $150 \mathrm{~cm}$ in height. It grows rapidly, resists browsing and can be used as a living fence along pastures; trimming it to $1.5 \mathrm{~m}$ height keeps foliage within reach of animals. Acacia farnesiana (L.) Willd (mimosa) is a thorny, fastgrowing bush. Its leaves are an excellent browse source despite the plant's high tannins content(11-16).

Forages are produced mainly as livestock feed. From a practical perspective, a forage's nutritional value depends principally on its content of soluble nutrients (minerals, protein, carbohydrates) and the degree of their availability(17). Mineral content in the two studied grasses was higher than in the legumes but lower than the 9.1-12.1\% reported for grasses from northeastern Mexico(18). Between the grasses, mineral values were very similar. In contrast, $A$. farnesiana had twice the $\mathrm{Ca}$ and $\mathrm{Zn}$ content, the lowest $\mathrm{K}$ content and the highest $\mathrm{Na}$ and $\mathrm{Mg}$ concentrations; $D$. virgatus and $G$. sepium had similar values.

Soil fertility and plant development both affect ash content. Forages produced with sufficient fertilization generally contain adequate mineral concentrations for use as a livestock feed. Mineral deficiencies or excesses in forage can cause deterioration in livestock health despite 
puede traer como consecuencia un bajo consumo voluntario del ganado y mayor tiempo de éstas en el rumen, lo que puede mejorarse con la inclusión de otras especies arbóreas en su dieta, incluyendo las estudiadas(20). Cabe mencionar que otros autores encontraron, para PC, datos que oscilan entre 13 a $23 \%$ en arbóreas tropicales(20-24), valores similares a los obtenidos en las leguminosas analizadas. En cuanto al extracto etéreo los valores son bajos, característico en los forrajes. Considerando los datos que reporta $\mathrm{FAO}(16)$ para el químico proximal de plantas empleadas como forrajes, reportan valores similares a los de este estudio.

Nutricionalmente el proceso de maduración es un factor importante que afecta los forrajes, los que están en crecimiento activo aun no maduro (con menor contenido de lignina) son los de mayor valor nutrimental. Durante la maduración de la planta se depositan concentraciones mayores de fibra lignificada provocando la disminución en el contenido de proteína y carbohidratos digestibles. El contenido de lignina, celulosa y hemicelulosa está relacionado con el grado de madurez de las plantas, Van Soest(25) y otros autores(17,26-27) asocian la maduración completa de las plantas con un alto contenido de FDA y lignina y baja concentración de celulosa y hemicelulosa. Esto se aplica a los datos obtenidos de las muestras estudiadas, donde Sorghum halepense (L.) Pers. tuvo una mayor madurez que Bothriochloa spp; mientras en las leguminosas el orden de madurez, de menor a mayor, fue: Desmanthus virgatus (L.) Willd, Gliricidia sepium (Jack.) Stend y Acacia farnesiana (L.) Willd.

Las panojas de gramíneas presentaron valores bajos en comparación con las vainas con semillas de las leguminosas en cuanto al contenido de lignina, en relación a la celulosa los intervalos fueron similares aunque en las leguminosas la Acacia farneciana fue la que tuvo el menor valor. En cuanto a las leguminosas, su etapa de maduración fue mayor que las gramíneas. Los valores más altos de apparently sufficient feed intake, leading to low production or reproduction in grazing ruminants. Mineral deficiencies can also cause extenuation, hair loss, skin disorders, non-infectious abortions, diarrhea, anemia, loss of appetite, bone abnormalities, low fertility and weight loss, among other clinical signs(19).

Crude protein (CP) content in the grasses was low. This could cause low voluntary intake by livestock and longer rumen residence time, although these could be improved by including arboreal species (like the legumes studied here) in the diet(20). For the legumes, CP was similar to previously reported values (13 to $23 \%$ ) for tropical legume trees(20-24). Ether extract levels were low in the present study, which is characteristic of forages. Overall, proximate analysis values observed in the present study were similar to those reported for forage plants(16).

Plant maturation affects the nutritional profile of forages. Actively growing plants that are not yet mature have lower lignin content and higher nutritional value. As plants mature, lignified fiber concentrations increase and protein and digestible carbohydrates contents decrease. Lignin, cellulose and hemicellulose contents are all related to plant maturation stage; indeed, Van Soest(25) and other authors(17,26-27) associate complete maturation in plants with high ADF and lignin contents, and low cellulose and hemicellulose levels. This is reflected in the studied plant species: $S$. halepense was more mature during the collection period than Bothriochloa spp.; D. virgatus was more mature than $G$. sepium which in turn was more so than A. farnesiana.

Lignin content was lower in the grass panicles than in the legume seedpods. Lignin is considered unassimilable by animals so the values observed here pose no problem for use in ruminant diets(29). Cellulose values did not differ among the tested plants, although $A$. farnesiana had the overall lowest value. Hemicellulose values were highest in the grasses 
hemicelulosa los presentaron las gramíneas, mientras que las vainas con semillas de leguminosas tuvieron un menor contenido. Éste se ve modificado por la edad de la planta y la variabilidad propia de la especie. Las muestras analizadas que tuvieron un mayor contenido de FDN y celulosa presentaron el menor contenido de proteína, lo que coincide con lo reportado por Van Soest(25) y Motta(28).

En cuanto a la lignina, cabe mencionar, que siendo un elemento considerado como no asimilable por los animales, los valores no representan inconvenientes para consumo de rumiantes(29).

El mayor contenido de sílice lo presentaron las gramíneas, sobresaliendo el Sorghum halepense, mientras que las leguminosas tuvieron valores similares. Ramírez et a/(30) mencionan que esta fracción tiene un efecto negativo sobre la digestibilidad de los polisacáridos de la pared celular, Van Soest(31) coincide con esta aseveración e indica que hay una interacción con la lignina. Los valores encontrados en este estudio no afectan la digestibilidad y consumo voluntario de los rumiantes, por lo que pueden ser consumidas libremente(29).

En cuanto a los factores antinutricios se menciona que estudios llevados a cabo en animales de laboratorio (ratas y pollos) demostraron que los inhibidores de tripsina interfieren con la digestión de proteínas, produciendo un incremento en la secreción pancreática e hipertrofia del mismo, dando como resultado una disminución en la tasa de crecimiento. Sin embargo, no se han reportado niveles críticos para rumiantes. Si se toma como referencia al frijol de soya crudo (588,000 UIT $\mathrm{g}^{-1}$ ) el contenido en las muestras analizadas es bajo(32).

El perfil de aminoácidos en $100 \mathrm{~g}$ de proteína fue similar en gramíneas y leguminosas, aunque el contenido de proteína fue mayor en las leguminosas. Esta información puede ser de utilidad en la formulación de dietas. El contenido and lowest in the legume seedpods. Maturation stage was more advanced in the legumes than in the grasses. These variables are modified depending on plant age and species; levels can even vary within a species. In the analyzed samples, those with higher NDF and cellulose contents also had lower protein content, which coincides with previous reports $(25,28)$.

Silica content was highest in the grasses, particularly $S$. halepense, and the legumes had values similar among the three species. This mineral has a negative effect on the digestibility of cell wall polysaccharides(30), and interacts with lignin(31). The present values for silica content would not affect digestibility or voluntary intake in ruminants, meaning they could be consumed freely(29).

Trypsin inhibitors interfere with protein digestion in rats and chickens under laboratory conditions, producing increased pancreatic secretion and hypertrophy, and resulting in decreased growth rate. Critical trypsin inhibitor levels have not been determined for ruminants, but if raw soy bean is used as a reference $(588,000$ TUI g-1) the content in the five studied plants is low(32).

The amino acids profile was similar among the five tested species, even though protein content was higher in the legumes. Hydrosoluble vitamin content was similar in the two plant groups. All the above data can be used in formulating balanced diets with these plants.

Chemical composition and nutritional quality in alternative forage plants like those tested here are influenced by genus, species and/or variety, soil type, climate and seasonal conditions during growth, maturation stage and plant element. Use of fertilizers, soil additives and irrigation water can also have an effect, although the tested plants were wild and therefore unaffected by these factors.

\section{CONCLUSIONS AND IMPLICATIONS}

The five unconventional forage plant species tested here are potential dietary elements for 
de vitaminas hidrosolubles fue similar en ambos grupos.

Los factores que pueden influir en la composición química y calidad nutricional de los alimentos no tradicionales como las plantas analizadas son género, especie o variedad, tipo de suelo en el que crecen, condiciones climáticas o estacionales durante el crecimiento, grado de maduración y parte de la planta; así como el uso de fertilizantes, enmiendas del suelo y el agua para riego. Este último factor no tuvo influencia en las muestras analizadas, ya que no son cultivadas.

\section{CONCLUSIONES E IMPLICACIONES}

Las cinco especies no convencionales estudiadas se pueden aprovechar como alimento para rumiantes cosechándolas y guardándolas como forraje y henificándolas como reserva de alimento en tiempos de sequía o crisis económica de los ganaderos. Se recomienda continuar con el estudio de estas plantas llevando a cabo pruebas de comportamiento animal (digestibilidad in vitro e in vivo).

\section{LITERATURA CITADA}

1. Viniegra G. Biotecnología para el aprovechamiento de los desperdicios orgánicos. Compilado por Monroy O y Viniegra G. AGT Editor S.A. México; 1981.

2. Audiffred PM. Fuentes no tradicionales de alimentos y su empleo en la alimentación de las aves de 1980 a 1986. Estudio recapitulativo [tesis licenciatura]. México, DF: Universidad Nacional Autónoma de México; 1988.

3. Villanueva SM. Fuentes no tradicionales de alimentos y su empleo en la alimentación de los ovinos de 1980-1987 [tesis licenciatura]. México, DF: Universidad Nacional Autónoma de México; 1989.

4. Church D. Basic animal nutrition and feeding. John Wiley and Sons, Inc. Vol. 1, 2th ed. Portland Book; 1987:31-47.

5. Almazán JM. Contribución al conocimiento de la flora arvense del Estado de Guerrero (México). Botánica Complutense 1993;(18):137-149.

6. AOAC. Official methods of analysis. 17th ed. Arlington, VA, USA: Association of Official Analytical Chemists. 2002.

7. Van Soest PJ. Analysis of forages and fibrous foods. Laboratory Manual of Animal Science. Cornell Univ. 1985. ruminants. They can be harvested and stored as forage and/or processed as hay to function as a feed reserve during drought and/or financial crisis. Further study of these potential forage species is needed to determine in vitro and in vivo digestibility.

End of english version

8. Kakade ML, Rackis JJ, McGhee JE, Puski G. Determination of trypsin inhibitors activity of soy-products. A collaborative analysis of an improved procedure. Cereal Chem 1974;(3):376-382.

9. Jaffé LA, Werner G, González ID. Isolation and partial Characterization of bean phytohemaglutinins. Phytochem 1974;(13):2685-2693.

10. Monroe EE, Wall E, Rolland ML. Detection and estimation of steroidal sapogenins in plant tissue. Annal Chem 1952;(24):1337-1341.

11. Gómez ME. Arbustos forrajeros utilizados en alimentación animal como fuente proteica. Colombia, CIPAV; 1955:1320.

12. Ackerman BA, Smith S. Las gramíneas de México. Tomo II. COTECOCA-SARH. 1987:344.

13. Flores MGA. Bromatología Animal. $3^{a}$ ed. México: Editorial Limusa; 1989.

14. Casas AJL. Etnobotánica mixteca. Sociedad, Cultura y Recursos naturales en la montaña de Guerrero. INICONACULTA; 1994:366.

15. Bogdan AV. Pastos tropicales y plantas de forraje. México: AGT Editor SA; 1997.

16. FAO, Animal Feed Resources Information System. http:// www.fao.org/ag/aga/frg/afris/es. Consultado 17 Feb, 2011.

17. Hughes, $\mathrm{HL}$, Heat ME, Metcalfe DS. Forrajes. México: Edit. C.E.C.SA. 1978.

18. Ramírez RG, Neira-Morales RR. Ruminal digestion characteristics and effective degradability of cell wall of browse species from northeastern Mexico. Small Ruminant Res 2000;(36):49-55.

19. Underwood, EJ. Trace elements in human and nutrition. 4th ed. USA: Academic Press; 1977.

20. López HMA, Rivera LJA, Ortega RL, Escobedo MJG, Magaña MMA, Sanginés GJR, Sierra VAC. Contenido nutritivo y factores antinutricionales de plantas nativas forrajeras del norte de Quintana Roo. Tec Pecu Méx 2008;(2):205-215.

21. Arellano ML, Carranco JME, Pérez-Gil RF, Hernández $P E$, Partida IH, Ripoll SH. Estudio de la composición química de 6 plantas no convencionales del Estado de Oaxaca, México, como recursos potenciales en la alimentación animal. Arch Lat Nutr 1993;(3):264-268.

22. Ortega CME, Carranco JME, Mendoza G, Castro G. Chemical composition of Guazuma ulmifolia Lam and its potential for ruminant feeding. Cuban J Agric Sci 1998;(4):283-286. 


\section{CARACTERIZACIÓN QUÍMICA DE ESPECIES FORRAJERAS NATIVAS PARA USO EN LA ALIMENTACIÓN ANIMAL}

23. Pinto R, Ramírez L, Vera K, Ortega L. Especies arbóreas y herbáceas forrajeras del sureste de México. Pastos y Forrajes 2002;(25):171-179.

24. Macías M, García A. Nota sobre la composición química de follaje de árboles de montaña del oeste de Cuba. Rev Compu Prod Porcina 2004;(2):41-45.

25. Van Soest PJ. Nutritional ecology of the ruminant. USA: Ed. O \& Books, Inc; 1982.

26. Hans, B. Elementos de nutrición animal. Zaragoza, España: Edit. Acribia; 1970.

27. Church DC, Pond WG. Basic animal nutrition and feeding. Portland, USA: O \& B Books; 1976.

28. Motta MS. Grasses and fodder resources in the British tropics. J Exp Agric 1952;(8):7-8.
29. Gutiérrez JL. Nutrición de rumiantes en pastoreo. Colección textos universitarios. Universidad Autónoma de Chihuahua. 1991.

30. Ramírez OR, Ramírez LRG, López GF. Factores estructurales de la pared celular del forraje que afectan su digestibilidad. Ciencia UANI, 2002;(2):180-189.

31. Van Soest PJ. Cell wall matrix interactions and degradation. In: Jung HG, Buxton DR, Hatfield RD, Ralph J editors. Forage cell wall structure and digestibility. ASA-CSSA-SSSA, Madison, WI. 1993;377-392.

32. Iwe MO, Ngoddy PO. Effect of extrusion on trypsin inhibitor contents of soy-sweet potato mixtures. J Food Process Preserv 2000;(6):453-463. 
